\title{
Home-Based Adaptation to Night-time Non-invasive Ventilation in Patients With Amyotrophic Lateral Sclerosis: A Randomised Controlled Trial Running Head: Home Adaptation to Non-invasive Ventilation in Amyotrophic Lateral Sclerosis
}

\author{
Eleonora Volpato ( $\nabla$ eleonora.volpato@unicatt.it ) \\ IRCCS Fondazione Don Carlo Gnocchi \\ Michele Vitacca \\ Istituti Clinici Scientifici Maugeri IRCCS, Respiratory Rehabilitation of the Institute of Lumezzane \\ Luciana Ptacinsky \\ IRCCS Fondazione Don Carlo Gnocchi \\ Agata Lax \\ IRCCS Fondazione Don Carlo Gnocchi \\ Salvatore D'Ascenzo \\ IRCCS Fondazione Don Carlo Gnocchi \\ Enrica Bertella \\ Istituti Clinici Scientifici Maugeri IRCCS, Respiratory Rehabilitation of the Institute of Lumezzane \\ Mara Paneroni \\ Istituti Clinici Scientifici Maugeri IRCCS, Respiratory Rehabilitation of the Institute of Lumezzane \\ Silvia Grilli \\ IRCCS Fondazione Don Carlo Gnocchi \\ Paolo Banfi \\ IRCCS Fondazione Don Carlo Gnocchi
}

\section{Research Article}

Keywords: Amyotrophic Lateral Sclerosis, ALS, Motor Neuron Disease, MND, Non-Invasive Ventilation, NIV, Home care

Posted Date: July 29th, 2021

DOI: https://doi.org/10.21203/rs.3.rs-631238/v1

License: (c) (i) This work is licensed under a Creative Commons Attribution 4.0 International License. Read Full License 


\section{Abstract}

Background: Adaptation to Non-Invasive Ventilation (NIV) in Amyotrophic Lateral Sclerosis (ALS) is generally implemented in an inpatient or outpatient setting.

Aims: To investigate whether adaptation to home-based NIV is as effective as outpatient one in ALS in terms of arterial carbon dioxide $\left(\mathrm{PaCO}_{2}\right)$ improvement. We also evaluated as secondary outcomes NIV acceptance, adherence and patient/caregiver satisfaction, quality of life (QoL) and caregiver burden.

Methods: Sixty-six ALS patients with indication for NIV were randomly assigned to two groups: 34 underwent NIV initiation at home (Home Adaptation, $\mathrm{HA}$ ) and 32 at multiple outpatient visits (Outpatient Adaptation, $\mathrm{OA}$ ). Respiratory function tests were performed at baseline (T0) together with blood gas analysis, which was repeated at the end of adaptation (T1) and after 2 and 6 months from T1. Overnight cardiorespiratory polygraphy was performed at T0, T2, and T3. NIV acceptance ( $\geq 5$ hours/night for 3 consecutive nights) and patient's and caregiver's expertise to manage NIV by an educational learning test were measured at T1; NIV adherence ( $\geq 150$ hours/month) was measured at T2 and T3. Short Form Health Survey (SF-36), Caregiver burden Inventory (CBI), Caregiver burden scale (CBS) and Zarit Burden Interview (ZBI) were performed at T0, T2 and T3.

Results: Fifty-eight participants completed the study. No significant differences were found between groups in $\mathrm{PaCO}_{2}$ at T1 $(p=0.46)$, T2 ( $p=0.50)$ and T3 $(p=0.34)$ as in acceptance $(p=0.55)$ and adherence to NIV at T2 and T3 ( $p=0.60$ and $p=0.75$, respectively). At T2, the patients' QoL, assessed with SF-36, was significantly better in HA than OA ( $p=0.01)$, but this improvement was not maintained up to T3 $(p=0.17)$.

Conclusions: In ALS, adaptation to NIV in the patient's home is effective as that performed in an outpatient setting, in terms of $\mathrm{PaCO}_{2}$ , acceptance and adherence. Improvement in quality of life was also found to be greater at home only after adaptation, opening the need for further studies to understand the role of environment with respect to NIV adherence.

\section{Introduction}

The most common cause of death in amyotrophic lateral sclerosis (ALS) is respiratory failure due to atrophy and weakness of the respiratory muscles. Diaphragmatic dysfunction can be the first manifestation or it can develop later as the disease progresses (1). The use of non-invasive ventilation (NIV) has markedly increased during the last two decades and is now an integral part of the management of both acute and chronic respiratory problems in different clinical conditions (2). It has been shown that patient compliance to NIV can slows pulmonary function decline in ALS (3), avoid or reduce the need for hospitalisation, improve quality of life (QoL), and lengthen survival (4-6). One study has recently shown that very early NIV initiation can improve survival in ALS patients (7). The study by Bertella et al. showed that outpatient NIV initiation is not inferior to inpatient NIV initiation in ALS, in terms of patients' acceptance and adherence (8).

Studies on home use of NIV have mostly focused on usage but not on the home as a setting for training and adaptation to NIV. Furthermore, the results have not always been noteworthy, probably due to poor initial monitoring opportunities, or because the studies were exclusively dealing with NIV modalities (9). Unfortunately, home-based NIV adaptation in ALS patients has not been sufficiently assessed as an option in standard care. It has not been established what the best setting for NIV adaptation is (i.e., hospital, outpatient clinic, or home, with or without tele-monitoring) (10-13). To our knowledge, there are no previous studies that demonstrated which is the best setting for NIV adaptation (i.e., outpatient clinic, hospital, home or telemonitoring) (10-13). Currently, there is a tendency to avoid hospitalization by promoting a different and less stressful approach for the ALS patients. Nevertheless, it is possible to detect differences across countries : while patient hospitalisation to initiate NIV in the United States is uncommon, in Europe, Japan or China the hospitalization remains the first choice $(10,12,13)$.

\section{Objectives}

The main aim of this study was to examine if home-based adaptation to NIV in ALS patients is as effective as that in the outpatient setting in terms of arterial carbon dioxide $\left(\mathrm{PaCO}_{2}\right)$ improvement. As a secondary aims we evaluated NIV acceptance and adherence, patient and caregiver satisfaction (satisfaction with NIV started in the two different settings), QoL, and the caregivers' perceived burden $(14,15)$. 


\section{Material And Methods}

This study was performed in accordance with the Helsinki Declaration. All participants provided their signed informed consent to take part in the study, which was approved by the Ethics Committee (on 15th April 2015); XXX. The registration ID at ClinicalTrials.gov is NCT02537132.

\section{Trial design and participants}

In this randomised controlled bicentric trial, we consecutively enrolled patients with ALS, diagnosed according to the revised EI Escorial criteria (16), who referred to the ALS outpatient clinics of the XXX and XXX between May 2015 and December 2017 for respiratory function assessment.

Inclusion criteria were age $\geq 18$ years with clinical indication for NIV according to EFNS criteria (2). Exclusion criteria were refusal to participate in the study; presence of severe cardiac/pulmonary comorbidity, as a contraindication to NIV; distance from hospital $>40$ $\mathrm{km}$ or other problems to reach the outpatient clinic; severe bulbar weakness and cognitive impairment that would preclude understanding the study protocol. This latter was ascertained using the Italian validated version of the Edinburgh Cognitive and Behavioural ALS Screen (ECAS) $(17,18)$. For clarity, the total score cut-offs were: 97 (age $\leq 60$ years, low-middle education) and 89 (age > 60 years, low-middle education); 108 (age $\leq 60$ years, high education) and 107 (age > 60 years, high education).

\section{Measures}

Data concerning age, sex, body mass index, spinal or bulbar onset, and time from ALS onset to NIV were collected.

At T0 (baseline) the patients underwent:

- - arterial blood gas analysis $(\mathrm{ABG})\left(\mathrm{pH}, \mathrm{PaCO}_{2}, \mathrm{paO}_{2}, \mathrm{HCO}_{3}\right)$ measured 4 hours after awakening;

- - clinical assessment, with the Revised Amyotrophic Lateral Sclerosis Functional Rating Scale (ALSFS-R) (19), Borg Dyspnoea Score (BDS) (20) and Epworth Sleepiness Scale (ESS) (21);

- - pulmonary function testing, including spirometry, performed in accordance with the European Respiratory Society guidelines, with the patient in a seated and supine position via a flanged mouthpiece, and using the suggested reference values (22), forced vital capacity (FVC), forced expiratory volume in the first second $\left(\mathrm{FEV}_{1}\right)$ and $\mathrm{FEV}_{1} / \mathrm{FVC}$ (Master Screen Body Jaeger Vyntus ${ }^{\mathrm{Tm}}$ Pneumo, Vyaire, Mettawa, IL, USA);

- - maximum inspiratory pressure (MIP) and maximum expiratory pressure (MEP) (MicroRPM Pressure Meter, Micro Medical Ltd, Lewiston, ME, USA) via a flanged mouthpiece, while the cheeks were held. Three measurements for a total of eight performed with less than $5 \%$ variability were recorded, and the highest value was used for the data analysis. (Patients with MEP $<60$ $\mathrm{cmH}_{2} \mathrm{O}$ were provided with a Cough Assist device) $(23,24)$;

- - overnight cardiorespiratory polygraphy (Embletta ${ }^{\text {TM }}$ PDS, Medcare, Iceland), according to the American Academy of Sleep Medicine clinical practice guidelines: apnoea and hypopnea were scored manually by standard criteria $(25,26)$;

- 36-item Short Form Survey (SF-36) (27), a questionnaire on the patient's health status;

-Caregiver Burden Inventory (CBI) (28), Caregiver Burden Scale (CBS) (29), Zarit Burden Interview (ZBI) (30), designed to detect the practical and psychological burden of the caregiver. These tools were used both at baseline and at the end of the study (6-month follow-up).

At T1 (after the 8-days adaptation period) the following evaluations were performed:

- - ABG measured 4 hours after awakening;

- - verification of NIV acceptance, i.e., a mean use of $\geq 5$ hours NIV per night for 3 consecutive nights during the adaptation trial;

- - a visual analogue scale (VAS), on which the patient indicated the degree of satisfaction with NIV management and nursing assistance (0-10, low-high);

- - educational learning test, designed to verify the knowledge and skills acquired by the patient with respect to the path taken with the physiotherapist (See Supplementary material-1). 
At T2 (2 months after T1) the patients underwent:

- - ABG measured 4 hours after awakening;

- - overnight cardiorespiratory polygraphy;

- - verification of adherence to NIV (nocturnal NIV usage for $\geq 150$ hours/month);

- - optimisation of the ventilator parameters (31);

- - VAS, SF-36, CBI, CBS, and ZBI (as above).

At T3 (6 months after T1) the following evaluations were performed:

- - ABG measured 4 hours after awakening;

- - overnight cardiorespiratory polygraphy;

- - verification of adherence to NIV;

- - BDS and ESS ;

- - VAS, SF-36, CBI, CBS, and ZBI.

Intervention: the NIV adaptation trial

Participants were randomized to the following groups:

- - Home Adaptation (HA): in addition to the usual medical care, patients received not less than 8 sessions at home with assistance provided by the Respiratory Therapist (RT) to help them adapt to NIV, and education on the management of bronchial secretions.

- - Outpatient Adaptation (OA): in addition to the usual medical treatment, patients attended not less than 8 sessions in the outpatient clinic to help them adapt to NIV and were educated about the management of bronchial secretions.

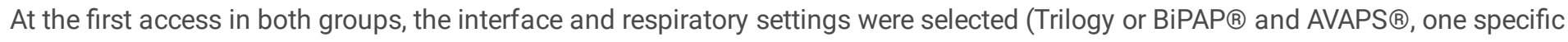
device that offers a bi-level ventilation mode allowing for application of an average tidal volume, Philips Respironics, Murrysville, PA, USA) in Spontaneous/Timed mode or Pressure Controlled mode with a pre-set tidal volume of $7 \mathrm{~mL} / \mathrm{Kg}$ and a fixed respiratory backup rate of 12 breaths/minute. At least 2-hours trial of NIV was conducted to monitor $\mathrm{SpO}_{2}$ and end tidal carbon dioxide $\left(\mathrm{ETCO}_{2}\right)$ using a CO2SMO monitor (Novametrix, Respironics, CA, USA). It provides reliable mainstream measurement and display of ETCO2 and respiratory rate under direct RT supervision. During this period, if $\mathrm{SpO}_{2}<94 \%$ or $\mathrm{ETCO}_{2}>45 \mathrm{mmHg}$, RT increased IPAP or EPAP until values normalized. A non-vented facial mask was connected to the CO2SMO probe and this latter to a whisper swivel (Koninklijke Philips Electronics N.V., Netherlands). Patients were recommended to use NIV only during the night and as much as possible until they had completely adapted to NIV. During the adaptation period, educational sessions were provided to each patient to ensure that the NIV use was adequate, and that the ventilator was being properly managed (max 8 sessions/patient). After each session, patients expressed their level of satisfaction by VAS. No inspired gas conditioning system was used during adaptation to NIV.

\section{Criteria for a correct adaptation to NIV}

The adaption to NIV was considered correct if the patient was able - with or without the caregiver's help - to put on the interface, manage the ventilator and the alarms, and clean the ventilator components.

The data obtained from the 2-hour monitoring was used to determine an average $\mathrm{SpO}_{2}>94 \%$ and average $\mathrm{ETCO}_{2}<45 \mathrm{mmHg}$ in patients with daytime hypoventilation (32).

The educational learning test given at the end of each session had to be passed (See Supplementary material-1). There was no objective difference between OA an HA in professional contact time and written information received. Our centres have a team of respiratory therapists who are specially trained in the NIV's initiation.

Criteria for acceptance of NIV

Page $4 / 15$ 
Patients were considered adapted to NIV and they could interrupt the trial when: 1) they used NIV $\geq 5$ hours/night for 3 consecutive nights verified by the data collected from the ventilator software; 2) patients, or caregivers, were able to accurately wear the mask, manage the ventilator and the alarms, and clean the ventilator components. On the other hand, the NIV 's adaptation was interrupted when 3) patients had failed to achieve NIV acceptance after 8 consecutive educational sessions.

\section{Sample size}

An initial power analysis was conducted using G*Power version 3.1.9.3 (33). We defined a non-inferiority margin of $0.4 \mathrm{kPa}(3.75$ $\mathrm{mmHg}$ ) for the difference in change of the primary endpoint, $\mathrm{PaCO}_{2}$, between home and outpatient initiation, as a difference less than $0.4 \mathrm{kPa}$ was meant to be clinically irrelevant. This statistical preliminary analysis was performed considering previous studies with NIV's $\mathrm{PaCO}_{2}$ changes of more than $0.45 \mathrm{kPa}$ (34-36). With a one-sided alpha of 0.05 , a beta of 0.2 , a Standard Deviation (SD) of 0.71 and expected drop-out rate of $25 \%$, at least 57.5 participants needed to be randomised.

\section{Randomisation, sequence generation and allocation concealment}

Eligible patients were allocated to HA or OA (i.e., home-based vs. outpatient clinic based NIV adaptation) using a method of minimisation, considering baseline bulbar function, baseline FVC, age and sex as the minimisation factors.

A centralised, web-based randomisation system was used to assign treatment allocation. A site-specific username and password were used to gain access to the system. Researchers were invited to enter patient details (identification number, date of birth and the minimisation factors) and to confirm consent and eligibility when completed. Then, the randomisation system notified the user and the study manager of the treatment allocation.

\section{Statistical analysis}

A complete statistical analysis plan was designed and approved before any analysis was carried out. Statistical analysis was performed using the Statistical Package for Social Science (SPSS, IBM® version 23). Socio-demographic data and clinical information were expressed as mean \pm standard deviation or as median and interquartile range. In order to compare the two groups and analyse interaction effects on outcome and process measures, analysis of variance (ANOVA) with two groups and three time periods were used. When there were significant interaction effects, t-tests were used to analyse the difference, pre and post in both groups. Similarly, between-group comparisons were performed using Welch's test for unequal variances. $\mathrm{H}_{0}$ was rejected if the under limit of the $95 \%$ confidence interval $(\mathrm{Cl})$ was less than the non-inferiority margin. Results were considered significant if $p<0.05$.

\section{Results}

Out of 82 ALS patients referred for evaluation for NIV initiation during the study period, 68 met the criteria to start NIV. Of these, two patients were pre-emptively initiated on NIV in the Intensive Care Unit because of an episode of acute respiratory failure. Therefore, 66 subjects started NIV and were randomised to HA $(n=34)$ or OA $(n=32)$.

The population was Italian-speaking, Caucasian, and mostly female (54.5\%). The baseline characteristics are illustrated in Table 1. 
Table 1

Baseline characteristics of the overall sample and both the experimental and the control group

\begin{tabular}{|c|c|c|c|c|}
\hline Characteristics & $\begin{array}{l}\text { Overall sample } \\
(n=66)\end{array}$ & $\begin{array}{l}\text { Home adaptation (HA) } \\
(n=34)\end{array}$ & $\begin{array}{l}\text { Outpatient Adaptation } \\
(\mathrm{OA})(\mathrm{n}=32)\end{array}$ & $\begin{array}{l}p \\
\text { value }\end{array}$ \\
\hline Female, n, \% & $36(54.5)$ & $14(41.2)$ & $16(50)$ & \\
\hline Age, y, mean (SD) & $69.1(8.6)$ & $67.4(7.5)$ & $70.9(9.5)$ & 0.11 \\
\hline BMI, mean (SD) & $24.09(6.7)$ & $23.4(2.9)$ & $24.7(4.3)$ & 0.15 \\
\hline Bulbar onset, n, \% & $19(28.8)$ & $7(20.6)$ & $12(37.5)$ & \\
\hline Spinal onset, n, \% & $43(65.1)$ & $25(73.5)$ & $18(56.2)$ & \\
\hline Respiratory onset, n, \% & $4(6.1)$ & $2(5.9)$ & $2(6.3)$ & \\
\hline $\begin{array}{l}\text { ALS duration (from symptom onset), } \\
\text { months, mean (SD) }\end{array}$ & $32(4.7)$ & $31.6(5.2)$ & $32.3(4)$ & 0.53 \\
\hline FVC (\% predicted) & $69.9(23.8)$ & 70 (23.09) & $70(21.4)$ & 0.52 \\
\hline $\mathrm{FEV}_{1}$ (\% predicted) & $68(24.3)$ & $72(22.8)$ & $64(25.8)$ & 0.20 \\
\hline $\mathrm{FEV}_{1} / \mathrm{FVC}$ (\% predicted) & $99.3(2.4)$ & $98.8(1.8)$ & $99.7(2.2)$ & 0.40 \\
\hline MIP (\% predicted) & $40.2(17.4)$ & $44.7(16.05)$ & $35.2(17.7)$ & 0.26 \\
\hline MEP (\% predicted) & $47.2(26.4)$ & $37.9(20.8)$ & $43.3(27.5)$ & 0.25 \\
\hline \multicolumn{5}{|l|}{ Ventilatory Mode, n, \% } \\
\hline S/T AVAPS & $30(45.5)$ & $13(38.2)$ & $17(53.1)$ & \\
\hline$S / T$ & $28(42.2)$ & $19(55.8)$ & $9(26.5)$ & \\
\hline PACV & $7(10.6)$ & $2(5.9)$ & $5(14.7)$ & \\
\hline APC/AVAPS & $1(1.5)$ & $0(0)$ & $1(2.9)$ & \\
\hline \multicolumn{5}{|l|}{ Kind of masks used, $n, \%$} \\
\hline Respironics non vented masks & $66(100)$ & $34(100)$ & $32(100)$ & \\
\hline Comfort Gel Blue Full & $25(37.9)$ & $14(41.2)$ & $11(34.4)$ & \\
\hline Easy Life & $18(27.3)$ & $9(26.5)$ & $9(28.1)$ & \\
\hline Amara Gel & $23(34.8)$ & $11(32.3)$ & $12(35.3)$ & \\
\hline ECAS, mean (SD) & $90.5(6.82)$ & $90.5(3.8)$ & $90(9.03)$ & 0.55 \\
\hline ALSFRS-R, mean (SD) & $29.2(6.6)$ & $28.7(6.4)$ & $29.8(6.9)$ & 0.53 \\
\hline BORG Dyspnoea Scale, mean (SD) & $0.49(0.5)$ & $0.39(0.8)$ & $0.59(0.31)$ & 0.19 \\
\hline ESS, mean (SD) & $9.2(0.4)$ & $9.8(0.3)$ & $9.4(0.4)$ & 0.25 \\
\hline
\end{tabular}

\section{-Table 1 should be inserted about here--}

A total of 58 participants completed the study. Overall, 4 participants (6.9\%), 2 in OA (6\%) and 2 in HA (5.9\%), did not reach the goal of 150 hours/month prescribed and 4 participants died (6.9\%), 2 in HA (5.9\%) and 2 in OA (6\%) and they withdrew from the study. Among the 4 participants who did not reach the 150 hours/month target, 2 (OA) did not perceive the need for NIV and rejected it, 1 (HA) was a bulbar onset patient who had trouble in using NIV for the sialorrhea problems and 1 (HA) preferred to use NIV only during the day (Fig. 1). No significant side effects were detected in the remaining ALS patients in the two study groups. 
Table 2 shows that the baseline differences between groups in terms of the ABG analysis and overnight cardiorespiratory polygraphy did not change significantly at follow-up. In both groups, $\mathrm{PaCO}_{2}$ significantly improved during the 2-months follow up, but not at 6months follow up.

Table 2

Arterial blood gas analysis and polygraphy between groups

\begin{tabular}{|c|c|c|c|c|c|c|c|c|c|c|c|c|}
\hline \multirow[t]{2}{*}{ Outcomes } & \multicolumn{3}{|c|}{ Baseline } & \multicolumn{3}{|c|}{ After adaptation } & \multicolumn{3}{|c|}{ Follow up (2 months later) } & \multicolumn{3}{|c|}{ Follow up (6 months later) } \\
\hline & $\begin{array}{l}\mathrm{HA} n= \\
34\end{array}$ & $\begin{array}{l}\mathrm{OA} \\
\mathrm{n}=32\end{array}$ & $p_{\text {value }}$ & $\begin{array}{l}\text { HAn } \\
=34\end{array}$ & $\begin{array}{l}O A \\
n=32\end{array}$ & $p_{\text {value }}$ & $\begin{array}{l}\mathrm{HA} \\
\mathrm{n}=31\end{array}$ & $\begin{array}{l}O A n= \\
27\end{array}$ & $p_{\text {value }}$ & $\begin{array}{l}\mathrm{HA} \\
\mathrm{n}=31\end{array}$ & $\begin{array}{l}\text { OA } n= \\
27\end{array}$ & $\begin{array}{l}\mathrm{p} \\
\text { value }\end{array}$ \\
\hline \multicolumn{13}{|l|}{$A B G$} \\
\hline $\begin{array}{l}\text { ph, mean } \\
\text { (SD) }\end{array}$ & $\begin{array}{l}7.39 \\
(1.27)\end{array}$ & $\begin{array}{l}7.42 \\
(0.44)\end{array}$ & $\begin{array}{l}p= \\
0.34\end{array}$ & $\begin{array}{l}7.39 \\
(2.8)\end{array}$ & $\begin{array}{l}7.39 \\
(3.1)\end{array}$ & $\begin{array}{l}p= \\
0.34\end{array}$ & $\begin{array}{l}7.39 \\
(3.45)\end{array}$ & $\begin{array}{l}7.41 \\
(0.02)\end{array}$ & $\begin{array}{l}p= \\
0.33\end{array}$ & $\begin{array}{l}7.42 \\
(0.02)\end{array}$ & $\begin{array}{l}7.40 \\
(14.6)\end{array}$ & $\begin{array}{l}p= \\
0.33\end{array}$ \\
\hline $\begin{array}{l}\mathrm{PaCO}_{2} \\
\text { mean (SD) }\end{array}$ & $\begin{array}{l}42.29 \\
(5.56)\end{array}$ & $\begin{array}{l}43.58 \\
(8.17)\end{array}$ & $\begin{array}{l}p= \\
0.46\end{array}$ & $\begin{array}{l}34.6 \\
(3.3)\end{array}$ & $\begin{array}{l}34.9 \\
(2.9)\end{array}$ & $\begin{array}{l}p= \\
0.46\end{array}$ & $\begin{array}{l}33.0 \\
(17.24)\end{array}$ & $\begin{array}{l}29.93 \\
(19.16)\end{array}$ & $\begin{array}{l}p= \\
0.50\end{array}$ & $\begin{array}{l}43.4 \\
(2.81)\end{array}$ & $\begin{array}{l}43.3 \\
(2.29)\end{array}$ & $\begin{array}{l}p= \\
0.46\end{array}$ \\
\hline $\begin{array}{l}\mathrm{PaO}_{2} \\
\text { mean (SD) }\end{array}$ & $\begin{array}{l}77 \\
(9.13)\end{array}$ & $\begin{array}{l}75.26 \\
(9.84)\end{array}$ & $\begin{array}{l}p= \\
0.46\end{array}$ & $\begin{array}{l}78.1 \\
(9)\end{array}$ & $\begin{array}{l}77.4 \\
(9.7)\end{array}$ & $\begin{array}{l}p= \\
0.46\end{array}$ & $\begin{array}{l}77.0 \\
(5.79)\end{array}$ & $\begin{array}{l}77.9 \\
(6.71)\end{array}$ & $\begin{array}{l}p= \\
0.61\end{array}$ & $\begin{array}{l}78.3 \\
(5.95)\end{array}$ & $\begin{array}{l}76.27 \\
(6)\end{array}$ & $\begin{array}{l}p= \\
0.24\end{array}$ \\
\hline $\begin{array}{l}\mathrm{HCO}_{3} \\
\text { mean (SD) }\end{array}$ & $\begin{array}{l}29.97 \\
(3.95)\end{array}$ & $\begin{array}{l}29.19 \\
(3.72)\end{array}$ & $\begin{array}{l}p= \\
0.80\end{array}$ & $\begin{array}{l}27.8 \\
(3.4)\end{array}$ & $\begin{array}{l}28.1 \\
(3.61)\end{array}$ & $\begin{array}{l}p= \\
0.28\end{array}$ & $\begin{array}{l}29.24 \\
(2.16)\end{array}$ & $\begin{array}{l}29.74 \\
(1.74)\end{array}$ & $\begin{array}{l}p= \\
0.37\end{array}$ & $\begin{array}{l}29.95 \\
(2.02)\end{array}$ & $\begin{array}{l}31.04 \\
(1.9)\end{array}$ & $\begin{array}{l}p= \\
0.11\end{array}$ \\
\hline $\begin{array}{l}\mathrm{SaO}_{2} \\
\text { mean (SD) }\end{array}$ & $\begin{array}{l}94.17 \\
(2.17)\end{array}$ & $\begin{array}{l}94.51 \\
(2.8)\end{array}$ & $\begin{array}{l}p= \\
0.59\end{array}$ & $\begin{array}{l}95.2 \\
(2.12)\end{array}$ & $\begin{array}{l}94.9 \\
(2.1)\end{array}$ & $\begin{array}{l}p= \\
0.21\end{array}$ & $\begin{array}{l}94.51 \\
(1.47)\end{array}$ & $\begin{array}{l}95.00 \\
(1.38)\end{array}$ & $\begin{array}{l}p= \\
0.24\end{array}$ & $\begin{array}{l}94.86 \\
(1.38)\end{array}$ & $\begin{array}{l}94.53 \\
(1.49)\end{array}$ & $\begin{array}{l}p= \\
0.44\end{array}$ \\
\hline \multicolumn{13}{|l|}{ Polygraphy } \\
\hline $\begin{array}{l}\text { AHI, mean } \\
\text { (SD) }\end{array}$ & $\begin{array}{l}13.34 \\
(11.71)\end{array}$ & $\begin{array}{l}19.99 \\
(17.96)\end{array}$ & $\begin{array}{l}p= \\
0.09\end{array}$ & - & - & - & $\begin{array}{l}5.90 \\
(4.99)\end{array}$ & $\begin{array}{l}6.65 \\
(5.83)\end{array}$ & $\begin{array}{l}p= \\
0.09\end{array}$ & $\begin{array}{l}5.20 \\
(4.57)\end{array}$ & $\begin{array}{l}7.11 \\
(7.45)\end{array}$ & $\begin{array}{l}p= \\
0.22\end{array}$ \\
\hline $\begin{array}{l}\mathrm{SpO}_{2} \\
\text { mean (SD) }\end{array}$ & $\begin{array}{l}91.91 \\
(2.13)\end{array}$ & $\begin{array}{l}90.69 \\
(3.51)\end{array}$ & $\begin{array}{l}p= \\
0.10\end{array}$ & - & - & - & $\begin{array}{l}91.8 \\
(5.2)\end{array}$ & $\begin{array}{l}92.1 \\
(6.1)\end{array}$ & $\begin{array}{l}p= \\
0.88\end{array}$ & $\begin{array}{l}91.60 \\
(16.25)\end{array}$ & $\begin{array}{l}91.43 \\
(16.76)\end{array}$ & $\begin{array}{l}p= \\
0.96\end{array}$ \\
\hline $\begin{array}{l}\text { ODI, mean } \\
\text { (SD) }\end{array}$ & $\begin{array}{l}11.47 \\
(10.36)\end{array}$ & $\begin{array}{l}11.10 \\
(7.52)\end{array}$ & $\begin{array}{l}p= \\
0.76\end{array}$ & - & - & - & $\begin{array}{l}8.6 \\
(3.8)\end{array}$ & $\begin{array}{l}8.15 \\
(3.23)\end{array}$ & $\begin{array}{l}p= \\
0.05\end{array}$ & $\begin{array}{l}4.85 \\
(4.91)\end{array}$ & $\begin{array}{l}6.57 \\
(8.20)\end{array}$ & $\begin{array}{l}p= \\
0.31\end{array}$ \\
\hline $\begin{array}{l}\text { T90\%, } \\
\text { mean (SD) }\end{array}$ & $\begin{array}{l}14.78 \\
(26.03)\end{array}$ & $\begin{array}{l}27.38 \\
(34.78)\end{array}$ & $\begin{array}{l}p= \\
0.10\end{array}$ & - & - & - & $\begin{array}{l}7.61 \\
(6.62)\end{array}$ & $\begin{array}{l}5.7 \\
(6.63)\end{array}$ & $\begin{array}{l}p= \\
0.07\end{array}$ & $\begin{array}{l}12.09 \\
(6.07)\end{array}$ & $\begin{array}{l}12.08 \\
(2.06)\end{array}$ & $\begin{array}{l}p= \\
0.14\end{array}$ \\
\hline
\end{tabular}

\section{-Table 2 should be inserted about here-}

Ten patients belonging to HA (29.4\%) complained of difficulties in adapting to NIV due to: more than 8 sessions required for the adaptation ( 3 patients, $8.8 \%$ ), pain or nose lesions (4 patients, $11.8 \%$ ), or low NIV's perceived need (3 patients, $8.8 \%$ ). These patients required two sessions more than the others to correctly adapt to NIV. On the other hand, 12 participants in OA (37.5\%) also needed more than 8 sessions to adapt to NIV. Nevertheless, no significant differences in terms of NIV hours of usage were found between groups $(F(1,42.3)=0.27, p=0.60)$ at T2 and $(F(1,40)=0.10, p=0.75)$ at 6-month follow-up (Fig. 2).

\section{-Figures 1 and 2 should be inserted about here-}

The rate of perceived exertion (BDS) at follow-up did not differ significantly between groups $(F(1,47.8)=0.54))$ at T3. Neither did daytime sleepiness, assessed by the ESS, differ significantly between groups at follow-up $(F(1,46.9)=0.15)$ at T3.

\section{Quality of life and caregiver burden}

At 2 months, QoL, assessed with the SF-36, improved more significantly in $\mathrm{HA}(F(1,55.82)=6.98, p=0.01)$ than in OA. This result, however, was not maintained over time, at 6 months follow-up $(F(1,1.94)=42.3, p=0.17)$.

Considering the perceived burden of caregivers and its effect on their QoL, assessed with the CBS, no differences were found between groups neither at $2(F(1,25)=1.29, p=0.27)$ and 6 months $(F(1,25)=0.00, p=0.93)$. However, significant differences were 
found within HA between T1 and T2 $(\mathrm{t}(17)=3.03, p=0.00)$ but not within OA $(\mathrm{t}(18)=2.08, p=0.05)$.

Physical, psychological, and social burden, examined with both the $\mathrm{CBI}$ and $\mathrm{ZBI}$, significantly improved only immediately after the adaptation process to NIV in OA (CBI: $p=0.01 ; \mathrm{ZBI}: p=0.00)$ but not in HA (CBI: $p=0.18 ; \mathrm{ZBI}: p=0.05)$.

Finally, the overall degree of satisfaction with the adaptation to NIV, measured by VAS at the end of the NIV adaptation, was significantly higher in HA than $\mathrm{OA}(\mathrm{F}(1,26)=7.48, p=0.01)$. However, this result was not maintained at $\mathrm{T} 2(\mathrm{~F}(1,59)=0.35, p=0.55)$ and T3 $(F(1,48)=0.43, p=0.51)$.

\section{Discussion}

Since 2015, in Italy, home-based adaptation to NIV has become an integral part of the care options for ALS patients, reserving the inpatient and outpatient settings for patients who are experiencing an acute decline, or who require multiple therapies, multidisciplinary diagnosis or need close nocturnal observation. Recent technological advances and the increased capability to remotely monitor ventilation have facilitated the use of the home's adaptation to NIV, where team and skills' experiences are relevant (37).

In our study, the primary outcome was $\mathrm{PaCO}_{2}$ equality between home and outpatient NIV adaptation and its maintenance over time. ABG was performed at least 4 hours after removing nocturnal ventilation to check whether the daytime hypoventilation state remained, demonstrating that there was no difference in $\mathrm{PaCO}_{2}$ values between the two groups over time. However, a significant difference $(p=0.02)$ was found between the $\mathrm{PaCO}_{2}$ measured at T2 compared with that at T3: this was due to the progression of the disease, although the patient increased the number of hours of NIV practiced during the day. However, this increases in ventilation hours at 6 months did not maintain $\mathrm{PaCO}_{2}$ at similar levels compared with those measured at T2. Dorst (10) showed that ventilation times naturally increase due to clinical worsening to the point of $24 \mathrm{~h}$ ventilation. In his paper, Markovic (38) demonstrated that ALS patients, after 3 months of adaptation to NIV, increased their ventilator use hours, demonstrating the natural progression of respiratory dysfunction as the disease worsened.

Perceptions of difficulties about NIV acceptance reported by patients were similar in the two groups. Our results are similar to those obtained by Chatwin et al. (39) in non-ALS neuromuscular patients, in whom outpatients increased their hours of nocturnal ventilation more than inpatients. In our study the acceptance of NIV during home adaptation was the same as the outpatients group. Our results show a high feasibility within the home NIV adaptation group compared to the outpatient adaptation group that needed more than 8 sessions in a higher percentage (29.4\% in HA vs. 37.5\% in OA). Moreover, ALS patients' adherence to NIV at 2 and 6 months indicate that outpatient and home-based initiation of NIV are equivalent: only 8 patients did not complete the study [4 participants (6.9\%), 2 in $\mathrm{OA}(6 \%)$ and 2 in $\mathrm{HA}(5.9 \%)$, did not reach the goal of 150 hours/month prescribed and 4 participants died (6.9\%), 2 in $\mathrm{HA}(5.9 \%)$ and 2 in $\mathrm{OA}(6 \%)]$.

Few studies investigated the effect of different ventilation modes and parameters on blood gases, clinical symptoms, and survival. Regarding the mode of ventilation, previous studies did not find significant differences between spontaneous/timed mode (S/T) and assisted pressure-controlled ventilation (aPCV). Furthermore, Sancho (40) showed that volume-preset ventilation is similar to pressure-preset ventilation in terms of survival, side-effects and lower NIV tolerance and Berlovitz showed that pressure assist-control ventilation is comfortable for the patient and it compensates for leaks (41). Crescimanno et al. (42) found that in neuromuscular disease with chronic ventilator failure, volume-assured pressure support NIV does not achieve better results than Pressure-preset NIV in terms of gas exchange, breathing patterns and comfort and it is associated with a higher rate of patient-ventilator asynchrony episodes. Dorst concluded that aPCV is the most commonly used mode in clinical practice (10). In our study, we used both the S/T and the aPCV mode with or without Average Volume-Assured Pressure Support (AVAPS), according to the continuous feedback of the patient to keep a breathing comfort.

There Are No Consensus Guidelines For NIV Regarding An Optimal Monitoring Strategy (43), adherence goals, or the best follow-up testing (43). In a prospective controlled study of ALS patients using NIV at home, Pinto et al. (11) demonstrated that NIV adjustment was successfully managed through telemonitoring and that NIV compliance was comparable to that of evaluated outpatients. This study stigmatizes how telemedicine improve survival and functional status in ALS patients and likely reduce disease costs. In our opinion, soon the telemedicine will play an important role in adherence verification and follow-up of home ventilated patients, but 
today there are no specific guidelines yet and more studies are needed to prove this argument. The home setting for NIV adaptation showed a greater improvement in the patients' QoL, but not in the caregiver's burden. Indeed, physical, psychological and social burden improved only in the $\mathrm{OA}$ and not in the HA, suggesting the importance of the perceived safety for the person who is constantly at the patient's side. In selecting which setting is most appropriate for NIV adaptation, it is important to consider factors such as the patient's transportation availability, distance from the hospital, the presence of a competent caregiver at night, severe bulbar weakness, and any anxiety or cognitive issues the patient may have. Patients who have the option of starting NIV at home are obviously in a more comfortable environment than in the hospital. Our home patients reported being in their own environment with the help of family and being able to sleep as much as needed, whereas patients seen in an outpatient setting were in an impersonal environment without the usual landmarks except caregiver support.

Our data are in line with the systematic review by Macintyre et al. (44) and the study of Bourke et al. (6) that showed a higher median survival for patients treated with NIV, together with an improved time-weighted mean HRQoL. However, they are in contrast with previous studies showing that the QoL of ALS patients tends to worsen, compared to the non-ALS group, after 6 months of NIV (45). On the other hand, as Hazenberg et al. noted in their observational study, methodological challenges regarding measurement of QoL over time in ALS patients because of disease progression that goes hand in hand with a change in QoL (45).

The results of our study indicate that patients who prefer OA or HA initiation may be allowed to choose. Factors to consider are the distance to travel to the referral center, whether they have cognitive or bulbar difficulties, anxiety, and the presence of a caregiver who is knowledgeable and familiar with the patient's needs.

\section{Limitations}

An accurate cost analysis was not performed in this study, but we can speculate that home-based NIV initiation is cost-effective considering that the Italian health system reimbursement for 1 day of hospitalization for an ALS patient in a rehabilitation centre is $€$ $370.37 /$ day and for 1 outpatient visit is $€ 230$ /day, while the reimbursement for home-based adaptation is $€ 47.00 /$ day. However, the costs of NIV are high, limiting the ability to extend the time of adaptation, especially given the out-of-pocket costs that are likely to significantly increase the economic burden. Indeed, Meng and colleagues found that monthly costs tend to increase nine months before diagnosis, with a significant increase in the index month (Medicare: \$10,398; commercial: \$9354), which persists post-indult. In addition, prescriptions and equipment costs are burdensome in the postdiagnosis period, reaching $70.2 \%$ of the annual cost trend due to disease progression $9 \%$ of total costs associated with the disease ( $\$ 126,161$ over the 10 years of disease duration) (46).

\section{Conclusions}

In ALS, adaptation to NIV at the patient's home is as effective as that performed in an outpatient setting, in terms of improved $\mathrm{PaCO}_{2}$, acceptance, and adherence. QoL seems better when NIV is offered at home, whereas there seems to be no benefit in terms of caregiver burden. HA was preferred in stable patients and their caregivers and was probably less costly. In addition, ALS patients need adequate follow-up to optimize treatment, adherence, and compliance.

\section{Abbreviations}

ALS = amyotrophic lateral sclerosis

NIV = non-invasive ventilation

QoL = quality of life

ALSFRS-R = Amyotrophic Lateral Sclerosis Functional Rating Scale-Revised

BDS= Borg Dyspnoea Scale

ECAS = Edinburg Cognitive and Behavioural ALS Screen

ARF = acute respiratory failure 


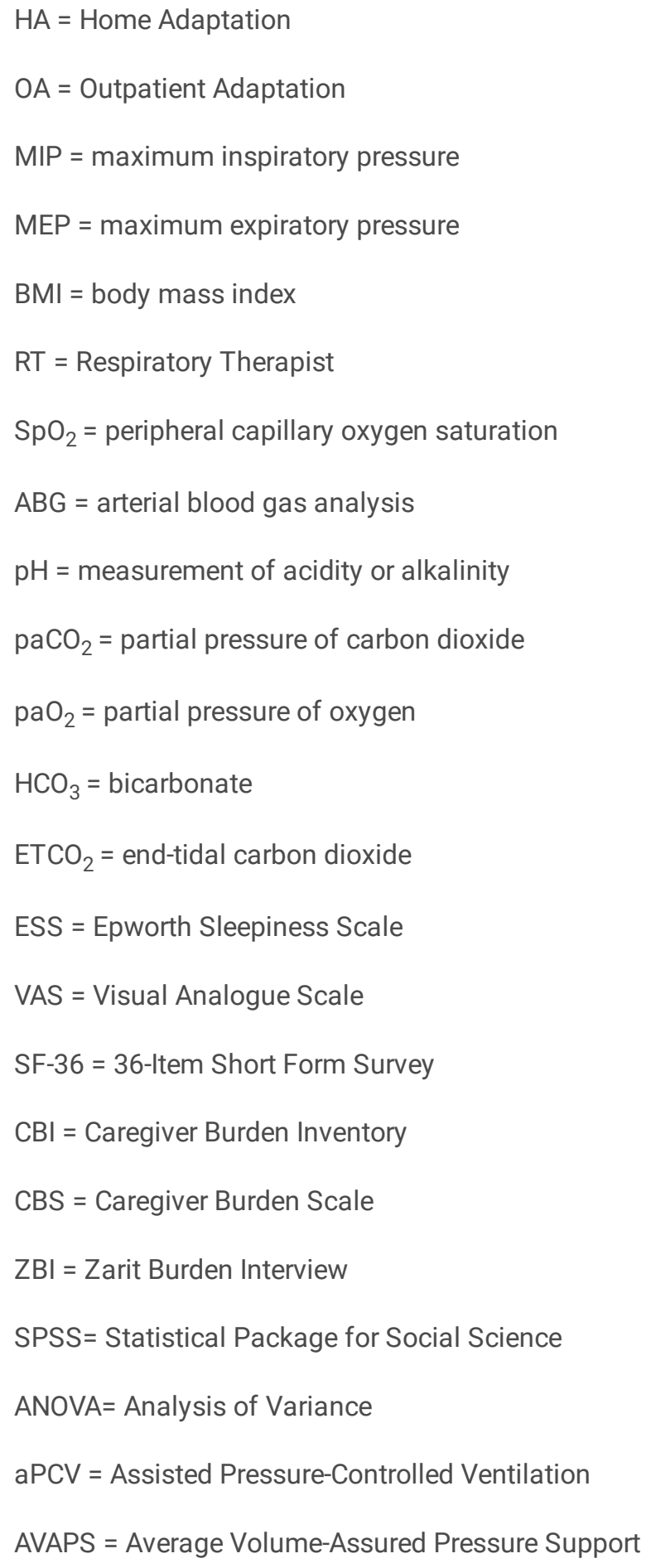

\section{Declarations}

\section{Acknowledgement}

The authors thank the participants, caregivers and the team at each site (Respiratory Rehabilitation Unit of the IRCCS Santa Maria Nascente, Fondazione Don Carlo Gnocchi, Milan; Respiratory Rehabilitation Unit of ICS Maugeri, Lumezzane, Brescia).

\section{Statement of Ethics}

All participants provided signed informed consent to take part in the study, which was approved by the Ethics Committee (approved on $15^{\text {th }}$ April 2015; Comitato Etico della Sezione IRCCS Fondazione Don Carlo Gnocchi, Board Affiliation: Comitato Etico IRCCS Regione Lombardia). The study was conducted according to the Declaration of Helsinky 2013. The requirements of the CONSORT checklist have been met. 
Conflict of interest statement

The authors declare that there is no conflict of interest.

\section{Consent for publication}

Informed consent was obtained from all individual participants included in the study. Written informed consent for publication of their clinical details was obtained from the patients.

\section{Funding}

The authors received no financial support for the research, authorship, and publication of this article.

\section{Author Contributions}

PB conceived and designed the study with MV. EV obtained ethics approvals, performed data analysis, prepared figures and tables, and drafted the manuscript, which was edited and approved by all authors. LP, SD, GS, EB and MP were leaders in recruiting participants and carrying out the intervention.

\section{Data Availability Statement}

All data generated or analysed during this study are included in this article [and/or] its supplementary material files. Further enquiries can be directed to the corresponding author.

\section{References}

1. Ricoy J, Rodríguez-Núñez N, Álvarez-Dobaño JM, Toubes ME, Riveiro V, Valdés L. Diaphragmatic dysfunction. Pulmonology. 2019;25(4):223-35.

2. Andersen PM, Abrahams S, Borasio GD, de Carvalho M, Chio A, Van Damme P, et al. EFNS guidelines on the clinical management of amyotrophic lateral sclerosis (MALS)-revised report of an EFNS task force. Eur J Neurol. 2012;19(3):360-75.

3. Kleopa KA, Sherman, M., Neal, B., Romano, G. J., \& Heiman-Patterson, T. Bipap improves survival and rate of pulmonary function decline in patients with ALS. J Neurol Sc. 1999;164(1):82-8.

4. Piepers S, van den Berg, J.P., Kalmijn, S., van der Pol, W.L., Wokke, J.H., Lindeman, E., \& van den Berg, L.H. Effect of non-invasive ventilation on survival, quality of life, respiratory function and cognition: a review of the literature. Amyotroph Lateral Scler. 2006;7(4):195-200.

5. Kaub-Wittemer D, Von Steinbuchel, N., Wasner, M., Laier-Groeneveld, G., Borasio, G.D. Quality of life and psychosocial issues in ventilated patients with amyotrophic lateral sclerosis and their caregivers. Journal of Pain and Symptom Management. 2003;26(4):890-6.

6. Bourke SC, Tomlinson, M., Williams, T.L., Bullok, R.E., Shaw, P.J., Gibson, G.J.. Effects of non-invasive ventilation on survival and quality of life in patients with amyotrophic lateral sclerosis: a randomized controlled trial. Lancet Neurol. 2006;5:140-7.

7. Vitacca M, Montini A, Lunetta C, Banfi P, Bertella E, De Mattia E, et al. Impact of an early respiratory care programme with noninvasive ventilation adaptation in patients with amyotrophic lateral sclerosis. Eur J Neurol. 2018;25(3):556-e33.

8. Bertella E, Banfi, P., Paneroni, M., Grilli, S., Bianchi, L., Volpato, E., Vitacca, M. Early initiation of night-time NIV in an outpatient setting: a randomized non-inferiority study in ALS patients. Eur J Phys Rehabil Med 2017;53(6):892-9.

9. $\mathrm{Kim} \mathrm{CH}$, \& Kim M.S.. Ventilator use, respiratory problems, and caregiver well-being in korean patients with amyotrophic lateral sclerosis receiving home-based care. J Neurosci Nurs. 2014;46(5):E25-32.

10. Dorst J, Ludolph AC. Non-invasive ventilation in amyotrophic lateral sclerosis. Ther Adv Neurol Disord. 2019;12:1756286419857040.

11. Pinto A, Almeida, J.P., Pinto, S., Pereira, J., Oliveira, A.G., De Carvalho, M. Home telemonitoring of non-invasive ventilation decreases healthcare utilisation in a prospective controlled trial of patients with amyotrophic lateral sclerosis.. $\mathrm{J}$ Neurol Neurosurg Psychiatry. 2010;81:1238-42. 
12. Sheers N, Berlowitz, D. J., Rautela, L., Batchelder, I., Hopkinson, K., \& Howard, M. E. Improved survival with an ambulatory model of non-invasive ventilation implementation in motor neuron disease. Amyotrophic Lateral Sclerosis and Frontotemporal Degeneration,. 2014;15(3-4):180-4.

13. Luján M, Moreno A, Veigas C, Montón C, Pomares X, Domingo C. Non-invasive home mechanical ventilation: effectiveness and efficiency of an outpatient initiation protocol compared with the standard in-hospital model. Respir Med. 2007;101(6):1177-82.

14. Chiò A, Gauthier, A., Calvo, A., Ghiglione, P., \& Mutani, R. Caregiver burden and patients' perception of being a burden in ALS. Neurology. 2005;64(10):1780-2.

15. Pagnini F, Rossi, G., Lunetta, C., Banfi, P., Castelnuovo, G., Corbo, M., \& Molinari, E. Burden, depression, and anxiety in caregivers of people with amyotrophic lateral sclerosis. Psychology, Health \& Medicine. 2010;15(685-693).

16. Ludolph A, Drory, V., Hardiman, O., Nakano, I., Ravits, J., Robberecht, W., \& Shefner, J. A revision of the El Escorial criteria-2015. Amyotroph Lateral Scler Frontotemporal Degener. 2015;16(5-6):291-2.

17. Abrahams S, \& Bak, T.. Edinburgh Cognitive and Behavioural ALS Screen-ECAS English version 2013. 2013.

18. Siciliano M, Trojano, L., Trojsi, F., Greco, R., Santoro, M., Basile, G., ... Monsurrò, M.. Edinburgh Cognitive and Behavioural ALS Screen (ECAS)-Italian version: regression based norms and equivalent scores.. Neurological Sciences. 2017;38(6):1059-68.

19. Cedarbaum JM, Stambler, N., Malta, E., Fuller, C., Hilt, D., Thurmond, B., ... A complete listing of the BDNF Study Group.. The ALSFRS-R: a revised ALS functional rating scale that incorporates assessments of respiratory function. Journal of the neurological sciences. 1999;169(1-2):13-21.

20. Just N, Bautin N, Danel-Brunaud V, Debroucker V, Matran R, Perez T. The Borg dyspnoea score: a relevant clinical marker of inspiratory muscle weakness in amyotrophic lateral sclerosis. Eur Respir J. 2010;35(2):353-60.

21. Johns MW. A new method for measuring daytime sleepiness: the Epworth sleepiness scale. Sleep. 1991;14(6):540-5.

22. Quanjer PH, Tammeling, G.J., Cotes, J.E., Pedersen, O.F., Peslin, R., Yernault, J.C. Lung volumes and forced ventilatory flows: report of Working Party “Standardization of Lung Function Test”. Eur Respir J 1993;6(16):5-40.

23. Chaudri MB, Liu C, Hubbard R, Jefferson D, Kinnear WJ. Relationship between supramaximal flow during cough and mortality in motor neurone disease. Eur Respir J. 2002;19(3):434-8.

24. Lalmolda C, Prados, H., Mateu, G., Noray, M., Pomares, X., Lujánab M.. Titration of Mechanical Insufflation-Exsufflation Optimal Pressure Combinations in Neuromuscular Diseases by Flow/Pressure Waveform Analysis. Archivos de Bronconeumología. May 2019;55(5):246-51.

25. Force. AAoSMT. Sleep-related breathing disorders in adults. Recommendations for syndrome definition and measurement techniques in clinical research. Sleep. 1999;22:667-89.

26. Berry RB, Budhiraja R, Gottlieb DJ, Gozal D, Iber C, Kapur VK, et al. Rules for scoring respiratory events in sleep: update of the 2007 AASM Manual for the Scoring of Sleep and Associated Events. Deliberations of the Sleep Apnea Definitions Task Force of the American Academy of Sleep Medicine. J Clin Sleep Med. 2012;8(5):597-619.

27. Brazier JE, Harper R, Jones NM, O'Cathain A, Thomas KJ, Usherwood T, et al. Validating the SF-36 health survey questionnaire: new outcome measure for primary care. BMJ. 1992;305(6846):160-4.

28. Novak M, Guest C. Application of a multidimensional caregiver burden inventory. Gerontologist. 1989;29(6):798-803.

29. Gupta R. The revised caregiver burden scale: a preliminary evaluation.. Research on Social Work Practice. 1999;9:508-20.

30. Chattat R, Cortesi V, Izzicupo F, Del Re ML, Sgarbi C, Fabbo A, et al. The Italian version of the Zarit Burden interview: a validation study. Int Psychogeriatr. 2011;23(5):797-805.

31. Gonzalez-Bermejo J, Perrin C, Janssens JP, Pépin JL, Mroue G, Leger P, et al. [Proposal for a systematic analysis of polygraphy or polysomnography for identifying and scoring abnormal events occurring during non-invasive ventilation]. Rev Mal Respir. 2014;31(4):312-22.

32. Kim SM, Park KS, Nam H, Ahn SW, Kim S, Sung JJ, et al. Capnography for assessing nocturnal hypoventilation and predicting compliance with subsequent noninvasive ventilation in patients with ALS. PLoS One. 2011;6(3):e17893.

33. Erdfelder E, Faul, F., \& Buchner, A.. GPOWER: A general power analysis program.. Behavior research methods, instruments, \& computers. 1996;28(1):1-11.

34. Hazenberg A, Kerstjens HA, Prins SC, Vermeulen KM, Wijkstra PJ. Initiation of home mechanical ventilation at home: a randomised controlled trial of efficacy, feasibility and costs. Respir Med. 2014;108(9):1387-95.

Page 12/15 
35. Duiverman ML, Vonk JM, Bladder G, van Melle JP, Nieuwenhuis J, Hazenberg A, et al. Home initiation of chronic non-invasive ventilation in COPD patients with chronic hypercapnic respiratory failure: a randomised controlled trial. Thorax. 2020;75(3):24452.

36. van den Biggelaar RJM, Hazenberg A, Cobben NAM, Gaytant MA, Vermeulen KM, Wijkstra PJ. A Randomized Trial of Initiation of Chronic Noninvasive Mechanical Ventilation at Home vs In-Hospital in Patients With Neuromuscular Disease and Thoracic Cage Disorder: The Dutch Homerun Trial. Chest. 2020;158(6):2493-501.

37. Gonzalez-Bermejo J, Morelot-Panzini, C., Arnol, N., Meininger, V., Kraoua, S., Salachas, F., et al.. Prognostic value of efficiently correcting nocturnal desaturations after one month of non-invasive ventilation in amyotrophic lateral sclerosis: a retrospective monocentre observational cohort study.. Amyotroph Lateral Scler Frontotemporal Degener. 2013;14:373-9.

38. Markovic N, Povitz M, Smith J, Leasa D, Shoesmith C, Gofton TE. Patterns of Non-Invasive Ventilation in Amyotrophic Lateral Sclerosis. Can J Neurol Sci. 2018;45(4):445-50.

39. Chatwin M, Nickol AH, Morrell MJ, Polkey MI, Simonds AK. Randomised trial of inpatient versus outpatient initiation of home mechanical ventilation in patients with nocturnal hypoventilation. Respir Med. 2008;102(11):1528-35.

40. Sancho J, Servera, E., Morelot-Panzini, C., Salachas, F., Similowski, T., \& Gonzalez-Bermejo, J. Non-invasive ventilation effectiveness and the effect of ventilatory mode on survival in ALS patients. Amyotrophic Lateral Sclerosis and Frontotemporal Degeneration. 2014;15(1-2):55-61.

41. Berlowitz DJ, Howard, M.E., Fiore, J.F.J., Vander Hoorn, S., O’Donoghue, F.J., Westlake, J., Smith, A., Beer, F., Mathers, S., Talman, P. Identifying who will beneft from non-invasive ventilation in amyotrophic lateral sclerosis/motor neurone disease in a clinical cohort.. J Neurol Neurosurg Psychiatry. 2016 Mar;87(3):280-6.

42. Crescimanno G, Marrone O, Vianello A. Efficacy and comfort of volume-guaranteed pressure support in patients with chronic ventilatory failure of neuromuscular origin. Respirology. 2011;16(4):672-9.

43. MD. ASSMaLW. Respiratory Care in Neuromuscular Diseases. Respir Care. 2018;63(5):601-8.

44. Maclntyre EJ, Asadi, L., Mckim, D. A., \& Bagshaw, S. M. Clinical outcomes associated with home mechanical ventilation: a systematic review. Canadian respiratory journal,. 2016;2016.

45. Hazenberg A, Kerstjens, H. A., Prins, S. C., Vermeulen, K. M., \& Wijkstra, P. J.. Is chronic ventilatory support really effective in patients with amyotrophic lateral sclerosis? Journal of neurology. 2016;263(12):2456-61.

46. Meng L, Bian A, Jordan S, Wolff A, Shefner JM, Andrews J. Profile of medical care costs in patients with amyotrophic lateral sclerosis in the Medicare programme and under commercial insurance. Amyotroph Lateral Scler Frontotemporal Degener. 2018;19(1-2):134-42.

\section{Figures}




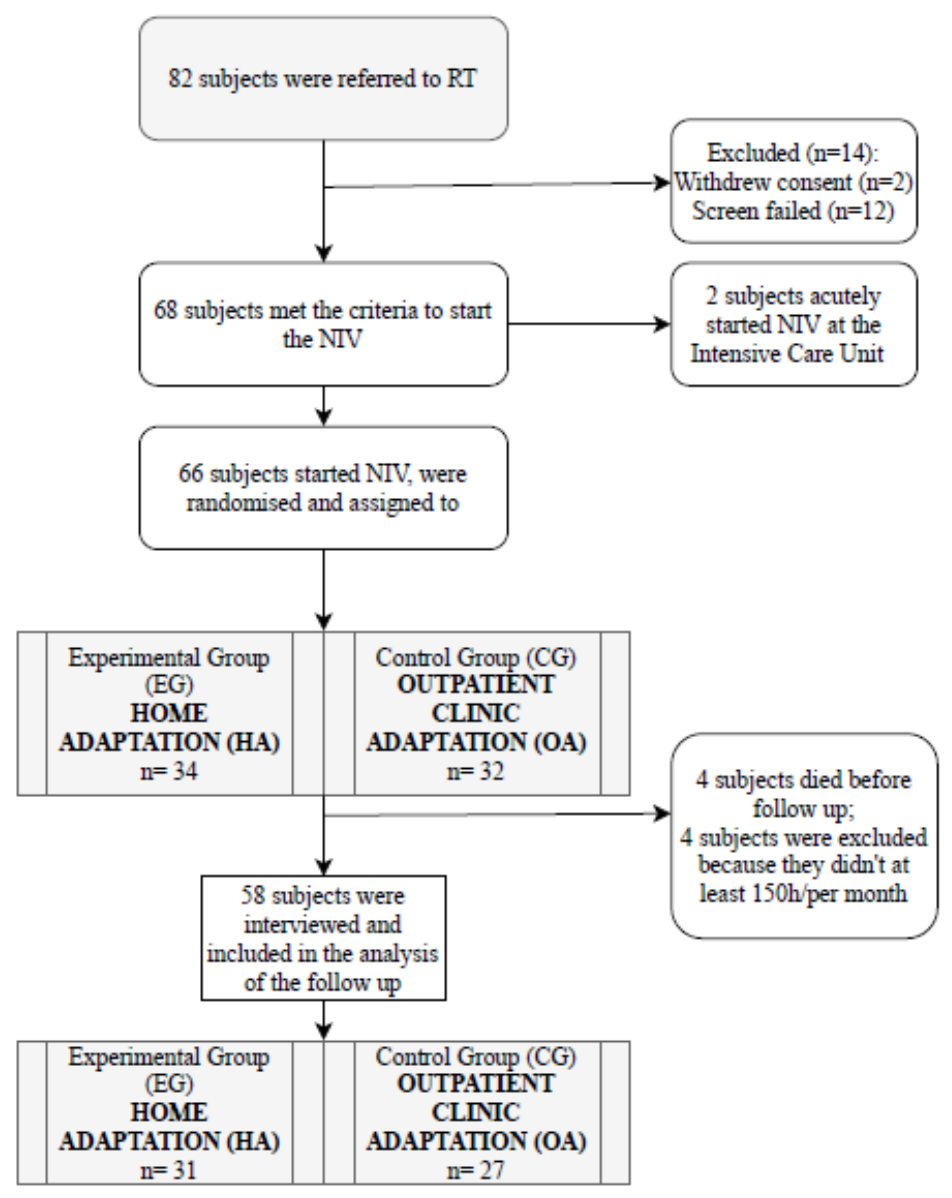

Figure 1

Flow chart of patient inclusion

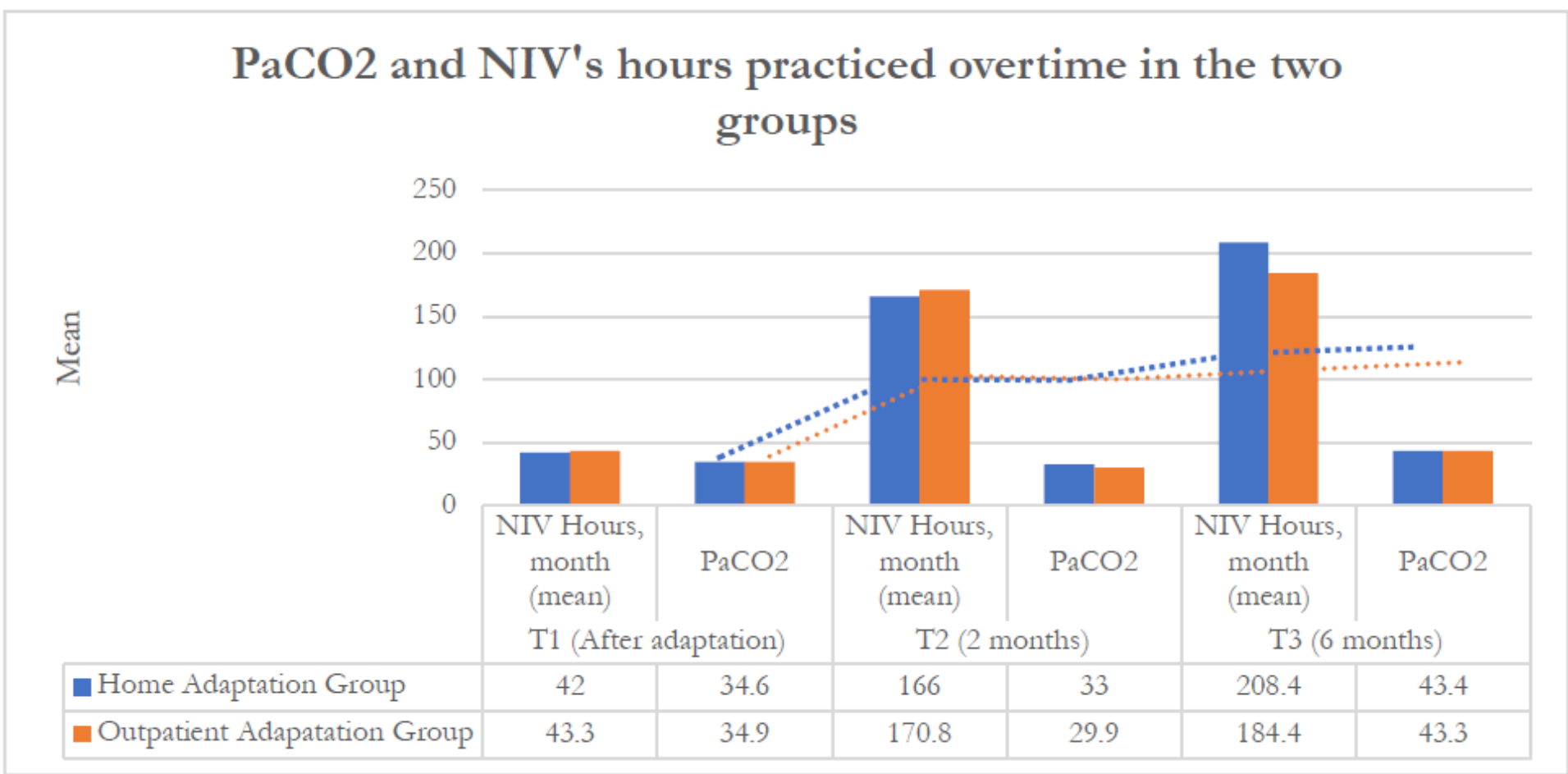

Figure 2 
PaCO2 and NIV adherence (hours of NIV used/month)

\section{Supplementary Files}

This is a list of supplementary files associated with this preprint. Click to download.

- Supplementarymaterials1Educationalformforcaregivers.xlsx 